University of Texas at El Paso

ScholarWorks@UTEP

3-1999

\title{
System Reliability: A Case When Fuzzy Logic Enhances \\ Probability Theory's Ability to Deal With Real-World Problems
}

Timothy J. Ross

Carlos M. Ferregut

The University of Texas at El Paso, ferregut@utep.edu

Roberto Osegueda

Vladik Kreinovich

The University of Texas at El Paso, vladik@utep.edu

Follow this and additional works at: https://scholarworks.utep.edu/cs_techrep

Part of the Computer Engineering Commons

Comments:

UTEP-CS-99-14.

Published in Proceedings of The 18th International Conference of the North American Fuzzy Information Society NAFIPS'99, New York City, June 10-12, 1999, pp. 81-84.

\section{Recommended Citation}

Ross, Timothy J.; Ferregut, Carlos M.; Osegueda, Roberto; and Kreinovich, Vladik, "System Reliability: A Case When Fuzzy Logic Enhances Probability Theory's Ability to Deal With Real-World Problems" (1999). Departmental Technical Reports (CS). 528.

https://scholarworks.utep.edu/cs_techrep/528

This Article is brought to you for free and open access by the Computer Science at ScholarWorks@UTEP. It has been accepted for inclusion in Departmental Technical Reports (CS) by an authorized administrator of ScholarWorks@UTEP.For more information, please contact Iweber@utep.edu. 


\title{
System Reliability: A Case When Fuzzy Logic Enhances Probability Theory's Ability to Deal With Real-World Problems
}

\author{
Timothy J. Ross \\ Department of Civil Engineering \\ University of New Mexico \\ Albuquerque NM 87131-1351, USA \\ ross@unm.edu \\ Carlos Ferregut, Roberto Osegueda, and Vladik Kreinovich \\ Future Aerospace Science and Technology Program (FAST) \\ Center for Structural Integrity of Aerospace Systems \\ University of Texas at El Paso \\ El Paso, TX 79968, USA \\ \{ferregut,osegueda,vladik\}@utep.edu
}

\begin{abstract}
In his recent paper "Probability theory needs an infusion of fuzzy logic to enhance its ability to deal with real-world problems", L. Zadeh explains that probability theory needs an infusion of fuzzy logic to enhance its ability to deal with real-world problems. In this talk, we give an example of a real-world problem for which such an infusion is indeed successful: the problem of system reliability.
\end{abstract}

\section{Reliability: Brief Introduction to the Problem}

\subsection{Reliability: general problem}

A typical system (e.g., an airplane) consists of several heterogeneous components. For a system to function normally, it is important that all these components function well.

For example, for an airplane to function normally, it is important that its structural integrity is intact, that its engines are functioning normally, that its communication system is functioning OK, and that the controlling software is functioning well.
The reliability of each component is normally analyzed by different engineering disciplines which use slightly different techniques. As a result of this analysis, we get the probabilities $f_{i}$ of each component's failure (or, equivalently, the probability $p_{i}=1-f_{i}$ that $i$-th component functions correctly). To estimates the reliability of the entire system, we must combine these probabilities that each component functions correctly into a single probability $p$ that the whole systems functions correctly.

\subsection{Damage assessment: a similar problem}

A similar problem occurs in damage assessment of engineering systems and structures. To find the damage, we make several measurements; for each component $x$, based on the result of each of these measurements, we can get a probability $f_{i}(x)$ that this component is defective. We want to make an assessment based on the result of all these measurements. Therefore, we must combine these probabilities into a single probability $f(x)$ that this component is faulty.

\subsection{Case study: structural integrity of aerospace structures}

One of the most important characteristics of the plane is its weight: every pound shaved off the plane 
means a pound added to the carrying ability of this plane. As a result, planes are made as light as possible, with their "skin" as thin as possible. However, the thinner the layer, the more vulnerable is the resulting structure to stresses and faults, and flight is a very stressful experience. Therefore, even minor faults in the plane's structure, if undetected, can be disastrous. To avoid possible catastrophic consequences, before the flight, we must thoroughly check the structural integrity of the plane.

Some faults, like cracks, holes, etc., are external, and can, therefore, be detected during the visual inspection. However, to detect internal faults (cracks, holes, etc.), we must somehow scan the inside of the thin plate that forms the skin of the plane. This skin is not transparent to light or to other electromagnetic radiation; very energetic radiation, e.g., X-rays or gamma-rays, can go through the metal, but it is difficult to use on such a huge object as a modern plane.

The one thing that easily penetrates the skin is vibration. Therefore, we can use sound, ultrasound, etc., to detect the faults. Usually, a wave easily glosses over obstacles whose size is smaller than its wavelength. Therefore, since we want to detect the smallest possible faults, we must choose the sound waves with the smallest possible wavelength, i.e., the largest possible frequency. This frequency is usually higher than the frequencies that we hear, so it corresponds to ultrasound.

Ultrasonic scans are indeed one of the main nondestructive NDE tools; see, e.g, [2, 3, 6, 17].

In nondestructive testing of structural integrity, we send an ultrasonic signal to the tested system, and measure the resulting vibration at different points. Our goal is to detect the points where the cracks or other possible faults are.

We would like to process this information as fact as possible. For large structures, however, with lots of sensors, and with a highly dynamical (ultrasonic) signal, we get a large amount of data, and processing this data as a whole would take too long. To decrease the data processing time, we can use the known fact that a vibration of a mechanical structure can be represented as a combination of different independent modes (corresponding to different eigenvalues of the corresponding matrix). Therefore, after measuring the vibrations, it is reasonable to separate the measurement results into results corresponding to different modes, and process each mode independently.

For each vibration mode, we can estimate the energy density at each point; if this measured energy density is higher than in the original (undisturbed) state, this is a good indication that a fault may be located at this point. The larger the increase in energy density, the larger the probability of a fault. As a result, for each point $x$, and for each mode $i$, we get the probability $f_{i}(x)$ that, based on the measurements related to this mode, there is a fault at a point $x$. We need to combine these probabilities into a probability $f(x)$ that there is a fault at $x$.

\subsection{Traditional approach to these problems}

The simplest case typically covered by statistical textbooks is when all components are independent. In this case:

- for reliability, the probability $p$ of the system's correct functioning is equal to the product of the correctness probabilities for components:

$$
p=p_{1} \cdot \ldots \cdot p_{n} .
$$

- for fault detection, the probability $p(x)=1-f(x)$ there there is no fault at a point $x$ is also equal to the product $p(x)=p_{1}(x) \cdot \ldots \cdot p_{n}(x)$ of the probabilities $p_{i}(x)=1-f_{i}(x)$ corresponding to different models, so, for $f(x)$, we get a formula $f(x)=1-\left(1-f_{1}(x)\right) \cdot \ldots \cdot\left(1-f_{n}(x)\right)$.

Another case with known answer is when all failures are caused by one and the same cause (case of full correlation). In this case, the failure probability is determined by its weakest link, so $f=\max \left(f_{1}, \ldots, f_{n}\right)$ and $p=\min \left(p_{1}, \ldots, p_{n}\right)$.

\subsection{Traditional approach is not always sufficient: a problem}

Most real-world situations lie in between these two extremes (see, e.g., $[12,13])$ :

- components are not completely independent (e.g., a structural fault can also damage sensors and thus, computational ability suffers), but

- components are not fully correlated.

Typically, we do not have sufficient information about the correlation between the components.

\section{Proposed Approach to Fusing Proba- bilities}

\subsection{Main idea}

We must choose a method for combining probabilities. In mathematical terms, we must describe, for every $n$, a function, which, takes $n$ numbers $p_{1}, \ldots, p_{n} \in$ 
$[0,1]$ as inputs and returns the "fused" (combined) probability $f_{n}\left(p_{1}, \ldots, p_{n}\right)$.

The division into components is rather subjective; e.g., for three components, we can

- either divide the system into these three subsystems 1,2 , and 3 ;

- or divide the system into two "macrocomponents" $\{1,2\}$ and 3 ,

- or divide it into 1 and $\{2,3\}$.

Depending on the division, we get different expressions for the resulting probability:

- If we divide the system into three components, the resulting probability is $p=f_{3}\left(p_{1}, p_{2}, p_{3}\right)$.

- If we divide the system into two macrocomponents $\{1,2\}$ and 3 , then for the first macrocomponent, we get $p_{12}=f_{2}\left(p_{1}, p_{2}\right)$, and thus, for a system as a whole, $p=f_{2}\left(p_{12}, p_{3}\right)=f_{2}\left(f_{2}\left(p_{1}, p_{2}\right), p_{3}\right)$.

- Similarly, if we divide the system into two macrocomponents 1 and $\{2,3\}$, then for the second macrocomponent, we get $p_{23}=f_{2}\left(p_{2}, p_{3}\right)$, and thus, for a system as a whole, $p=f_{2}\left(p_{1}, p_{23}\right)=$ $f_{2}\left(p_{1}, f_{2}\left(p_{2}, p_{3}\right)\right)$.

The resulting probability should not depend on the (subjective) subdivision into components. As a result, we should get $f_{3}\left(p_{1}, p_{2}, p_{3}\right)=f_{2}\left(f_{2}\left(p_{1}, p_{2}\right), p_{3}\right)=$ $f_{2}\left(p_{1}, f_{2}\left(p_{2}, p_{3}\right)\right)$. In other words, we can make two conclusions:

- First, the combination function for arbitrary $n>2$ can be expressed in terms of a combination function corresponding to $n=2$, as $f_{n}\left(p_{1}, \ldots, p_{n}\right)=$ $f_{2}\left(p_{1}, f_{2}\left(p_{2}, \ldots, f_{2}\left(p_{n-1}, p_{n}\right) \ldots\right)\right)$.

- Second, the function $f_{2}$ which describes the combination of two probabilities should be associative (i.e., $f_{2}\left(a, f_{2}(b, c)\right)=f_{2}\left(f_{2}(a, b), c\right)$ for all $a, b, c \in[0,1])$.

Associativity is a reasonably strong property, but associativity alone is not sufficient to determine the operation $f_{2}(a, b)$, because there are many different associate combination operations. Hopefully, there is another property which we can use. To describe this property, let us recall that for reliability, the function $g_{2}\left(p_{1}, p_{2}\right)$ has the following meaning:

- $p_{1}$ is the probability $P\left(C_{1}\right)$ of the event $C_{1}$ defined as "the first component is functioning correctly";
- $p_{2}$ is the probability $P\left(C_{2}\right)$ of the event $C_{1}$ defined as "the first component is functioning correctly"; and

- $f_{2}\left(p_{1}, p_{2}\right)$ is the probability $P\left(C_{1} \& C_{2}\right)$ that both components are functioning correctly.

In some reliability problems, several components serve as back-ups for one another; in such situations, the system as a whole functions correctly if at least one of the components functions correctly. In other words, in such problems, we are interested in the value

$P\left(C_{1} \vee C_{2}\right)$. From the probability theory, we know that $P\left(C_{1} \vee C_{2}\right)=P\left(C_{1}\right)+P\left(C_{2}\right)-P\left(C_{1} \& C_{2}\right)$, i.e., $P\left(C_{1} \vee C_{2}\right)=p_{1}+p_{2}-f_{2}\left(p_{1}, p_{2}\right)$. We can describe this expression as $P\left(C_{1} \vee C_{2}\right)=g_{2}\left(p_{1}, p_{2}\right)$, where $g_{2}(a, b)=$ $a+b-f_{2}(a, b)$.

Similar to the case of "and"-combination of different components, we can describe the probability $P\left(C_{1} \vee C_{2} \vee C_{3}\right)$ (that are least one of three components functions correctly) in two different ways:

- either as $P\left(\left(C_{1} \vee C_{2}\right) \vee C_{3}\right)=g_{2}\left(g_{2}\left(p_{1}, p_{2}\right), p_{3}\right)$,

- or as $P\left(C_{1} \vee\left(C_{2} \vee C_{3}\right)\right)=g_{2}\left(g_{2}\left(p_{1}, p_{2}\right), p_{3}\right)$.

The expression for $P\left(C_{1} \vee C_{2} \vee C_{3}\right)$ should not depend on how we compute it, and therefore, we should have $g_{2}\left(g_{2}\left(p_{1}, p_{2}\right), p_{3}\right)=g_{2}\left(p_{1}, g_{2}\left(p_{2}, p_{3}\right)\right)$. In other words, not only the function $f_{2}(a, b)$ should be associative, but also the function $g_{2}(a, b)=a+b-f_{2}(a, b)$ should be associative.

\subsection{Resulting solution}

Functions $f(a, b)$ for which both the function itself and the expression $a+b-f(a, b)$ are associative have been classified in 1979 [5]. These functions (called Frank's t-norms) are described by a formula

$$
f_{2}(a, b)=\log _{s}\left[1+\frac{\left(s^{a}-1\right) \cdot\left(s^{b}-1\right)}{s-1}\right]
$$

for some constant $s$. As a particular case of this general formula, we get the above two expressions $a \cdot b$ (for $s \rightarrow 1$ ) and $\min (a, b)$ (for $s \rightarrow 0$ ).

\subsection{Connection with fuzzy techniques}

In the above text, we described everything in terms of combining probabilities. However, from the mathematical viewpoint, the resulting requirements were exactly the requirements traditionally used for combining membership values in fuzzy approach (see, e.g., $[8,9]$ ): 
- The function $f_{2}\left(p_{1}, p_{2}\right)$ which describes the degree of truth of the statement $C_{1} \& C_{2}$, provided that we know the degrees of truth for statement $C_{1}$ and $C_{2}$, is a $t$-norm.

- The function $g_{2}\left(p_{1}, p_{2}\right)$ which describes the degree of truth of the statement $C_{1} \vee C_{2}$, provided that we know the degrees of truth for statement $C_{1}$ and $C_{2}$, is a t-conorm.

Moreover, it is in the context of combining fuzzy values that the above Frank's result was first obtained.

Fuzzy techniques have been successfully used in nondestructive testing in particular (for a latest survey, see, e.g., [4]) and in damage assessment in general (see, e.g., $[15,16])$.

The fact that for probabilistic data, we get similar formulas, makes us hope that this algebraic approach will be able to combine probabilistic and fuzzy data.

\section{Case Study: Results}

As a case study, we applied the new method to the problem of non-destructive evaluation of structural integrity of Space Shuttle's vertical stabilizer. To prove the applicability of our method, we applied this techniques to measurement results for pieces with known fault locations. The value $s$ was determined experimentally so as to achieve the best performance; it turned out that the best value is $s \approx 1$ (corresponding to the independent modes).

For this value $s$, our method detected all the faults in $\approx 70 \%$ of the cases, much larger proportion than with any previously known techniques (for details, see $[1,10,11,14])$. For other values of $s$, we got an even better detection, but at the expense of false alarms.

\section{Acknowledgments}

This work was supported in part by NASA under cooperative agreement NCC5-209, by NSF grants No. DUE-9750858 and CDA-9522207, by the United Space Alliance, grant No. NAS 9-20000 (PWO C0C67713A6), by the Future Aerospace Science and Technology Program (FAST) Center for Structural Integrity of Aerospace Systems, effort sponsored by the Air Force Office of Scientific Research, Air Force Materiel Command, USAF, under grant number F4962095-1-0518, and by the National Security Agency under Grant No. MDA904-98-1-0564.

\section{References}

[1] G. Andre, Comparison of Vibrational Damage Detection Methods in an Aerospace Vertical Stabilizer Structure, Master Thesis, The University of Texas at El Paso, Civil Engineering Department, May 1999.

[2] D. E. Chimenti, "Guided waves in plates and their use in materials characterization", Appl. Mech. Rev., 1997, Vol. 50, No. 5, pp. 247-287.

[3] R. W. Clough and J. Penzien, Dynamics of Structures, McGraw Hill, N.Y., 1986.

[4] C. Ferregut, R. Osegueda, and A. Nunez (eds.), Proceedings of the International Workshop on Intelligent NDE Sciences for Aging and Futuristic Aircraft, El Paso, TX, September 30-October 2, 1997.

[5] M. J. Frank, "On the simultaneous associativity of $F(x, y)$ and $x+y-F(x, y)$ ", Aequationes Mathematicae, 1979, Vol. 19, No. 2-3, pp. 194-226.

[6] I. Grabec and W. Sachse, Synergetics of Measurement, Prediction, and Control, Springer Verlag, BerlinHeidelberg, 1997.

[7] X. E. Gros, NDT Data Fusion, J. Wiley, London, 1997.

[8] G. Klir and B. Yuan, Fuzzy sets and fuzzy logic: theory and applications, Prentice Hall, Upper Saddle River, NJ, 1995.

[9] H. T. Nguyen and E. A. Walker, A first course in fuzzy logic, CRC Press, Boca Raton, Florida, 1997.

[10] R. A. Osegueda, A. Revilla, L. Pereyra, and O. Moguel, "Fusion of modal strain energy differences for localization of damage", In: A. K. Mal (ed.), Nondestructive Evaluation of Aging Aircraft, Airports, and Aerospace Hardware III, Proceedings of SPIE, Vol. 3586, Paper 3586-28.

[11] L. R. Pereyra, R. A. Osegueda, C. Carrasco, and C. Ferregut, "Damage detection in a stiffened plate using modal strain energy differences", Ibid, Paper 3586-29.

[12] H. Petroski, Design paradigms: case histories of error and judgment in engineering, Cambridge University Press, Cambridge, MA, 1994.

[13] T. J. Ross, "Case studies in civil engineering: fuzzy logic applications", In: B. Papadopoulos and A. Syropoulos (eds.), Current Trends and Developments in Fuzzy Logic, Proceedings of the First International Workshop, Thessaloniki, Greece, October 16-20, 1998 (to appear).

[14] N. S. Stubbs, T. Broom, and R. A. Osegueda, "Nondestructive construction error detection in large space structures", AIAA ADM Issues of the International Space Station, AIAA, Williamsburg, Virginia, April 1998, pp. $47-55$

[15] T. Terano, K. Asai, and M. Sugeno, Fuzzy Systems Theory and its Applications, Academic Press, San Diego, CA, 1987.

[16] M. Ulieru and R. Isermann, "Design of a fuzzy-logic based diagnostic model for technical processes", Fuzzy Sets and Systems, 1993, Vol. 52, No. 3, pp. 249-272.

[17] I. A. Viktorov, Rayleigh and Lamb Waves: Physical Theory and Applications, Plenum Press, N.Y., 1967. 